IASSNS-HEP-97/118

hep-th/9710219

October 1997

\title{
Matrix Models and String World Sheet Duality
}

\author{
S. P. de Alwis由户 \\ School of Natuaral Sciences, Institute for Advanced Study, Princeton NJ 08540
}

\begin{abstract}
The scaling limits used recently to derive matrix models, and a certain analyticity assumption, are invoked to argue that the agreement between some matrix model calculations and supergravity is a consequence of string world sheet duality.
\end{abstract}

\footnotetext{
${ }^{1}$ e-mail: dealwis@sns.ias.edu, dealwis@gopika.colorado.edu
}

${ }^{2}$ On leave from Department of Physics, Box 390, University of Colorado, Boulder, CO 80309. 
In a recent paper Seiberg[1]] has given a derivation of the matrix model[2]. However there appears to be some confusion as to whether the argument in [1] effectively bypasses checks on whether gauge theory calculations agree with supergravity. The problem stems from the fact that neither in the light-like version nor in the space-like version of the matrix model has the connection to the supergravity effective action been directly established as in the case of string theory. In the latter case, as is well known, the consistency conditions for the propagation of strings results in a background which obeys the equations of supergravity with also a systematic prediction as to what the higher derivative corrections to Einstein's equations are. There is no such demonstration in the case of the matrix model, indeed it has not even been put in a covariant form. Hence we believe some further clarification, even if only in a certain limited area, of the relationship between matrix model calculations and supergravity is of some interest. To this end we will give an argument using the limit considered in [1] t an assumption of analyticity, and string theory world sheet duality, to establish that, at least for processes with one impact parameter and for which longitudinal (i.e. 11th direction) momentum transfer is zero, the matrix model reproduces supergravity.

The action for $N$ Dp-branes involves in general higher derivative terms and multiple commutatator terms of the gauge fields $(A)$ living on the brane. However in the 'gauge theory' limit [1], [3] [4]

$$
l_{s} \equiv \sqrt{\alpha^{\prime}} \rightarrow 0, \quad g \rightarrow 0, \text { with } g_{m}^{2}=(2 \pi)^{p-2} g l_{s}^{p-3}, A_{\mu}, \text { fixed }
$$

( $l_{s}$ is the string scale, $g$ is the string coupling and $A$ is the gauge field ) one gets the matrix model action,

$$
S=-\frac{1}{4 g_{m}^{2}} \int_{W_{p+1}} \operatorname{tr}\left(F_{\alpha \beta} F^{\alpha \beta}+D_{\alpha} X^{i} D^{\alpha} X_{i}+\left[X^{i}, X^{j}\right]^{2}\right) .
$$

Note that the indices $\alpha, \beta$ are those tangential to the p-brane and $i, j$ are transverse to

\footnotetext{
${ }^{1}$ This limit has also been used by Sen[3] to give a uniform description of matrix models and also by J. Maldacena四 to discuss the relation to supergravity calculations. In fact our discussion will use some of the results of the latter paper.
} 
it. The fields were identified in terms of the ten-dimensional gauge fields in the usual fashion, i.e. $F_{\alpha \beta}=\left[D_{\alpha}, D_{\beta}\right], D_{\alpha}=\partial_{\alpha}-A_{\alpha}, X_{i}=A_{i}, F_{\alpha i}=D_{\alpha} X_{i}, F_{i j}=\left[X_{i}, X_{j}\right]$. These fields are $U(N)$ matrices and the expression for the action includes a $U(N)$ trace.

In the rest of this note we will confine ourselves to zero-branes. It is expected that considerations involving other branes can be obtained in a similar manner. However it is important to point out that in the limit (0.1) only one kind of brane survives. Thus in 10 dimensions one chooses $p=0$ and only zero-branes will survive with all other branes being composites of those. This is of course part of the derivation of matrix models discussed in [1], [3]. Thus our arguments will be valid only in these maximally supersymmetric situations.

In matrix model calculations of the forces between branes one integrates out fluctuations around a classical background configuration corresponding to the relative positions of the branes [2]. Consider $N$ D0 branes with one of them being treated as a probe brane separated from the others (which are coincident) by a distance (impact parameter) $b$ along the 2-axis and moving with a velocity $v$ along the 1 -axis.

Thus we put $X^{i}=B^{i}+Y^{i}$, where the background gauge field $B^{1}$ has the element $v t / l_{s}^{2}$ on the upper left-hand corner and zeros everywhere else and $B^{2}$ has $b / l_{s}^{2}$ at the upper left-hand corner and zero everywhere else. The important point is that the 'gauge theory' limit in which $B$ is held fixed as $l_{s} \rightarrow 0$ corresponds to holding $v / l_{s}^{2}$ and $b / l_{s}^{2}$ fixed. The term in the effective lagrangian coming from the L-loop gauge theory diagram with $I$ insertions of the 'velocity' background field $F \equiv \dot{B}^{1}$ then takes the form

$$
c_{I, L}(N) g_{m}^{2 L-2} \frac{F^{I}}{X^{3 L+2(I-2)}}=c_{I, L}(N) \frac{F^{2}}{g_{m}^{2}}\left(\frac{g_{m}^{2} F^{2}}{X^{7}}\right)^{L}\left(\frac{F}{X^{2}}\right)^{I-2 L-2}
$$

This is just the standard loop expansion in the large $\mathrm{N}$ limit with the factors of $X \equiv B^{2}$ inserted by dimensional analysis. (See [5] [四], and references therein.)

Let us now consider the string theory calculation of this effective action to arbitrary order in string perturbation theory. Firstly in this limit since $g \rightarrow 0$, all handles (corresponding to string creation and annihilation) are suppressed. Thus at any order one has an integrand with a product of terms corresponding to cylinderical world sheets attached 
to a disc with factors of the form

$$
e^{-t\left(k^{2}+M^{2}\right)}
$$

Here $t$ is some Schwinger parameter and $M^{2}=M_{B}^{2}+M_{F}^{2}+M_{g}^{2}$ is the squared mass operator, with the different terms on the right hand side being the bosonic fermionic and ghost contributions. Explicitly we have [7]

$$
\begin{aligned}
M_{B}^{2} & =\left(\frac{b}{2 \pi l_{s}^{2}}\right)^{2}+i \frac{\epsilon}{l_{s}^{2}} N_{0}^{+}+\frac{i \epsilon(1-\epsilon)-2 a_{B}}{l_{s}^{2}} \\
& +\frac{1}{l_{s}^{2}} \sum(\text { non }- \text { zero modes }) .
\end{aligned}
$$

(where $\pi \epsilon=\tanh ^{-1} v$ and $N_{0}^{+}$is a zero mode in a light-like direction)) with similar expressions for the fermions and the ghosts. It should be stressed that this whole calculation makes sense only in the superstring context [7]. The purely bosonic contribution would diverge at $v=0$ so that the velocity expansion would make no sense. Thus although we will not be making explicit use of supersymmetry the fact that we are dealing with the superstring seems essential to our considerations. In effect the argument indirectly implies the existence of a supersymmetric non-renormalization theorem.

In the 'gauge theory' limit $\left(l_{s} \rightarrow 0 b / l_{s}^{2}, \epsilon / l_{s}^{2} \rightarrow v / l_{s}^{2}\right.$ fixed), the only surviving (nonconstant) contribution to the mass operator is

$$
M^{2} \rightarrow\left(\frac{b}{2 \pi l_{s}^{2}}\right)^{2}+\frac{i v}{l_{s}^{2}}\left(N_{0}^{+}-N_{0}^{+}(R)\right)
$$

where $N_{0}, N_{0}(R)$ are certain zero modes coming from the bosonic and Ramond sectors [7]. The point is that all the massive open string states drop out in the gauge theory limit and only the BPS states survive?

The effective lagrangian of the probe brane as computed from open string theory has the general form

$$
L\left(X, F, g_{m}^{2}, l_{s}\right)=\sum_{I, L} c_{I, L}\left(N, l_{s} X\right) \frac{F^{2}}{g_{m}^{2}}\left(\frac{g_{m}^{2} F^{2}}{X^{7}}\right)^{L}\left(\frac{F}{X^{2}}\right)^{I-2 L-2} .
$$

\footnotetext{
${ }^{2}$ As this paper was being prepared for publication a paper which also contains this observation appeared[6]
} 
In the limit $l_{s} \rightarrow 0$ keeping $X, F, g_{m}$ fixed this must, by the previous argument, reduce to the 'gauge theory' expression (0.3) so that $c_{I, L}(N, 0)=c_{I, L}(N)$ where the right hand side is the coefficient in the gauge theory.

Now consider the region $l_{s} X=\frac{b}{l_{s}}>1$ where the effective action can be computed in terms of a supergravity action which will contain an infinite series of higher derivative terms. The effective action in question is that of a brane propagating in the corresponding supergravity background. The action of such an object may be written as

$$
S=-\frac{1}{g l_{s}} \int d t e^{-\phi} \sqrt{\operatorname{det} g}+\frac{1}{l_{s}} \int C
$$

where the zero mode of the dilaton has been explicitly factored out and the last term is the coupling of the R-R field. From the closed string point of view as long as the background curvature is small $\left(l_{s}^{2} R<<1\right)$ which is the case for large $l_{s} X>1$, there is a meaningful expansion for $g, \phi . C$ that is consistent with closed string propagation in powers of $l_{s}$. These fields will be solutions of the all orders in $l_{s}$ expansion in supegravity Lagrangians. In terms of the original parameters $F$ and $X$ however this version of the effective action must have the same expasion as (0.7) except that the coefficients $c_{I, L}\left(N, l_{s} X\right)$ are now replaced by $c_{I, L}^{S G}\left(N, l_{s} X\right)$ the superscript $S G$ denoting the fact that they are to be computed from the (infinite series of) supergravity actions. Now in the region $l_{s} X>1$ one has from the usual closed string argument, $c_{I, L}\left(N, l_{s} X\right)=c_{I, L}^{S G}\left(N, l_{s} X\right)$. Now we have to make a crucial assumption that these functions are analytic. Clearly since the supergravity expansion must break down at $l_{s} X=1$ there must be a pole at this value on the real axis. However there is no reason to expect any cuts etc which would prevent the analytic continuation of this equation to the region inside the circle $\left|l_{s} X\right|=1$ and in particular to the point $l_{s} X=0$. Thus assuming analticity we have the result,

$$
c_{I, L}(N)=c_{I, L}(N, 0)=c_{I, L}^{S G}(N, 0)
$$

where the first equality was established earlier.

For the diagonal terms $(I=2 L+2)$ the coefficient $c_{I, L}^{S G}(N, 0)$ can be calculated from the lowest order in $l_{s}$ supergravity action. This is because on dimensional grounds the 
higher derivative terms will not contribute to these terms [5]. Thus if one puts in the explicit classical supergravity solution [8], [4, we get

$$
S=-\frac{1}{(2 \pi)^{2} g_{m}^{2} l_{s}^{4}} \int d t f^{-1}\left(\sqrt{1-f v^{2}}-1\right)
$$

where

$$
f=1+\frac{k}{l_{s}^{4}}, \quad k \equiv \frac{c g_{m}^{2} N}{X^{7}}
$$

with c a known constant. Now taking the gauge theory limit one gets (see for example [4] and references therein)

$$
L\left(X, F, g_{m}^{2}, l_{s}=0\right)=-\frac{1}{g_{m}^{2}} k^{-1}\left(\sqrt{1-k F^{2}}-1\right) .
$$

But by our previous argument and in particular equation (0.9) this is the same object that was calculated in the gauge theory. Hence the two expressions must be the same. This appears to be a generalization of the old result that the closed string (and hence classical gravity) appears as a quantum effect in open string perturbation theory. As has been pointed out in [2] this object has an M-theory interpretation. In particular as shown in [5] the metric dialton and RR field in (0.12) can be regarded as being obtained from the null reduction of a M-theory metric due to a supergraviton source.

It is useful at this point to discuss the units in which various physical quantities in the theory are being defined. While a choice of units is obviously not going to change the physics, a convenient choice will clarify the aspects of the physics that we wish to study better than some other choice. Since what seems to emerge from the study of matrix models is 11-D supergravity it is natural to set the (classical) eleven dimensional Newton constant equal to one. (This is particularly useful if one wishes to study quantum effects around classical solutions). With the velocity of light being set equal to one also, we keep Planck's constant $\hbar=l_{P}^{9}$. i.e. the parameter that defines the semi-classical (quantum loop) expansion is the ninth power of the Planck length. This is the natural system of units to use in any discussion of quantum (semi-classical) corrections (such as Hawking radiation) to solutions of classical gravitational field equations (such as black holes). We 
then have the following formulae.

$$
\begin{gathered}
16 \pi G_{N}=1, c=1, \quad \hbar \equiv l_{P}^{9}, \quad l_{m}^{-3} \equiv\left(2 \pi g_{m}\right)^{2} \\
g^{2}=\frac{l_{P}^{3}}{l_{m}^{3}}=\frac{\hbar^{\frac{1}{3}}}{l_{m}^{3}}, \quad l_{s}=l_{P}^{\frac{1}{2}} l_{m}^{\frac{1}{2}}=\hbar^{\frac{1}{18}} l_{m}^{\frac{1}{2}} \\
\frac{b}{l_{P}}=X l_{m}=\frac{x_{m}}{l_{m}}, \quad \frac{v}{l_{P}}=F l_{m}
\end{gathered}
$$

The above formulae clarify the relation between the matrix model quantum mechanics (characterized by the Yang-Mills length scale $l_{m}$ ) and the 11D or target space quantum mechanics characterized by $l_{P} \equiv \hbar^{\frac{1}{9}}$. The limit considered in [1], [3], 四, corresponds to taking the dimensionless ratio $\frac{l_{P}}{l_{m}}$ to zero. As one sees from (0.3) the condition for the validity of the loop expansion in Yang-Mills quantum mechanics is that the dimensionless number $l_{m} X=\frac{b}{l_{P}}>>1$. In other words measured in 11D Planck units the impact parameter must be large, although measured in string units it is going to zero.

The rescaling to gauge theory variables (and the above choice of units) also clarifies the issue of whether higher derivative terms in the 11-D supergravity action (which should be there on general grounds as quantum corrections - see for example [9]) are reproduced by matrix model calculations. At first sight the answer seems to be negative. Let us write the semi-classical expansion (which in our units is the same the low-energy expansion) of the action as

$$
\begin{aligned}
I & =\frac{1}{l_{P}^{9}} \int \sqrt{g} R+\ldots \\
& +\frac{1}{l_{P}^{7}} \int d^{11} \sqrt{g} " R^{2} "+\ldots
\end{aligned}
$$

In the above the first line is the classical 11-D action while the second line denotes all possible $R^{2}$ terms etc coming from quantum effects. The limit $l_{P} \rightarrow 0$ in this action would just appear to pick up the leading classical term which would seem to imply that the matrix model just gave only classical supergravity. However we also need to rescale the coordinates (corresponding to the last line of (0.13) such that

$$
x \rightarrow x_{m}=l_{m}^{2} X=\frac{l_{m}}{l_{P}} x .
$$


Since the limit is taken with $l_{m}$ fixed this means that small distances in the original variables become large distances in the new variable. Thus the action is now

$$
\begin{aligned}
I & =\frac{1}{l_{m}^{9}} \int d^{11} x_{m} \sqrt{g} R_{m}+\ldots \\
& +\frac{1}{l_{m}^{7}} \int d^{11} x_{m} \sqrt{g} " R^{2}{ }_{m}+\ldots
\end{aligned}
$$

Thus the quantum expansion parameter is now $l_{m}$ which is fixed and the matrix model is expected to pick up all the higher order terms. Thus any non-vanishing non-diaganol terms in the matrix model calculation should correspond to the higher-derivative terms in the supergravity effective action. The two theories one with Planck's constant $l_{P}^{9}$ the other with Planck's constant $l_{m}^{9}$ appear to be equivalent to the two theories, the auxiliary one with Planck mass $\tilde{M}_{P}$ and the other with Planck mass $M$, introduced by Seiberg [1].

We should stress however that the actual argument made above applies only to zero momentum transfer processes in the 11 direction (since it depends on string theory arguments). We believe that this is consistent with the argument in [1] that the finite $\mathrm{N}$ light-like compactified M-theory is equivalent to the limit (0.1) of string theory. It seems to us therefore that further calculations such as that of Polchinski and Pouliot 10 provide non-trivial checks of the validity of the matrix model.

Before we conclude we should stress that the arguments of this paper can be interpreted as just a statement about ten-dimensional string theory (as in [11]) without any reference whatsoever to [2] and the subsequent developments. Thus one can think about the result as being just a statement relating the calculation of a D0-brane effective action from two different perpectives. Obviously however they are of current interest because of the possible connection to M-theory that was first pointed out in [2].

Finally we should comment on two papers [12, [6] that appeared as this note was being prepared for publication. The first involves the scattering of three gravitons to three gravitons and so it depends on two impact parameters. The arguments above apply explicitly only to the one impact parameter situation. In particular on the closed string side of the argument we have used analyticity in impact parameter space (which 
is equivalent to analycity in the complex angular momentum place (5). In the two impact parameter case the singularity structure is clearly more complicated it is possible that this analytic continuation is invalid. Similar remarks would apply to the relation of this work to [6] where the matrix theory is tested on an ALE space.

Acknowledgments: I would like to thank Dan Kabat and Nati Seiberg for useful discussions and comments on the manuscript. I'm also grateful to, Finn Larsen, Joe Polchinski, and Ashoke Sen for comments which led me to clarify the assumptions behind my argument and to V.P. Nair and Peter Orland and Tom Banks for comments on the relevance of Regge poles and cuts. I would also like to thank Edward Witten for hospitality at the Institute for Advanced Study and the Council on Research and Creative Work of the University of Colorado for the award of a Faculty Fellowship. This work is partially supported by the Department of Energy contract No. DE-FG02-91-ER-40672.

\section{References}

[1] N. Seiberg, "Why is the Matrix Model Correct?" hep-th/9710009.

[2] T. banks, W. Fischler, S. Shenker, L. Susskind, Phys. Rev. D55 (1997) 5112; hepth/9610043.

[3] A. Sen, "D0 Branes on $T^{n}$ and Matrix Theory" hep-th/9709220.

[4] J. Maldacena "Branes Probing Black Holes" hep-th/9709099.

[5] K. Becker, M. Becker, J. Polchinski and A. Tseytlin, "Higher Order Graviton Scattering in M(atrix) Theory", hep-th/9706072.

[6] M. Douglas and H. Ooguri, "Why Matrix Theory is Hard" hep-th/9710178.

[7] C. Bachas Phys. Lett. B374, 37 (1996) hep-th/9511043.

[8] G. Horowitz and A. Strominger, Nucl. Phys. B360, (1991) 197.

\footnotetext{
${ }^{3}$ I'm grateful to V.P. Nair for pointing this out to me.
} 
[9] M.B. Green, M. Gutperle and P. Vanhove, Phys. Lett. B409 (1997) 177, hepth/970175

[10] J. Polchinski and P. Pouliot, "Membrane Scattering and M-momentum transfer" hep-th/9704029.

[11] M. Douglas, D. Kabat, Pouliot and S. Shenker, Nucl. Phys. B485 (1997) 85 hepth/9608024.

[12] M. Dine and A. Rajaraman, "Multigraviton Scattering in the Matrix Model", hepth/9710174. 